\title{
Zn $\begin{array}{ll}\text { Research Square } & \begin{array}{l}\text { They should not be considered conclusive, used to inform clinical practice, } \\ \text { or referenced by the media as validated information. }\end{array}\end{array}$
}

\section{Molecular Insights into Tumor Targeted Therapy by Carrier-Free Lycorine Nanoparticles in Vitro and in Vivo}

\section{Shiyue Zhou}

Tianjin University of Traditional Chinese Medicine

\section{Yuling Qiu}

Tianjin Medical University

Rui Liu

Institute of Biomedical Engineering, Chinese Academy of Medical Sciences \& Peking Union Medical

College

\section{Shuangshuang Yin}

Tianjin University of Traditional Chinese Medicine

\section{Yingying Shao}

Tianjin University of Traditional Chinese Medicine

\section{Song Wu}

Tianjin University of Traditional Chinese Medicine

\section{Qian Chen}

Tianjin University of Traditional Chinese Medicine

\section{Tao Wang}

Tianjin University of Traditional Chinese Medicine

\section{Bo Du}

Institute of Biomedical Engineering, Chinese Academy of Medical Sciences \& Peking Union Medical College

\section{Haiyang Yu ( $\nabla$ hyyu@tjutcm.edu.cn )}

Tianjin University of Traditional Chinese Medicine

\section{Research}

Keywords: Lycorine, carrier-free nanoparticle, apoptosis, autophagy, metastasis

Posted Date: June 29th, 2021

DOI: https://doi.org/10.21203/rs.3.rs-651393/v1 
License: (c) (i) This work is licensed under a Creative Commons Attribution 4.0 International License. Read Full License 


\section{Abstract}

Lycorine (Ly) is a promising natural compound with extensive antitumor activities. However, due to poor aqueous solubility and low bioavailability, the research and development of Ly anticancer drugs has been greatly restricted. Recently, the carrier-free nanoparticles have emerged as an outstanding drug delivery system. In this study, we prepared a DSPE-PEG modifying Ly nanoparticle (DS-Ly NPs) to overcome these drawbacks. We find that compared with Ly, DS-Ly NPs can significantly inhibit cell viability. Meanwhile, DS-Ly NPs treatment results in higher promotion of apoptotic and autophagic capacities, as well as inhibition of migration and invasion. Furthermore, DS-Ly NPs exhibits a better antitumor effect in AOM/DSS induced colorectal tumors and orthotopic hepatocellular tumors than Ly in repression of multiple oncogenic processes, which may benefit from the advantages of carrier-free nanoparticle. Beside, we showed that DS-Ly NPs can prolong the blood circulation time better than Ly. Taken together, these results have demonstrated that carrier-free nanoparticle provides a new therapeutic strategy for treating cancer.

\section{Introduction}

In recent years, a lot of attention has been paid to the active ingredients in traditional herbal medicine, which have played an important role in the fight against diseases for thousands of years [1-3]. More and more active components of Traditional Chinese Medicine have been found and confirmed [4-6].

Paclitaxel, artemisinin and other drugs have also been widely recognized as first-line treatment drugs for related diseases $[2,7]$. However, the molecular pharmacological mechanism of many active components has not been fully elucidated. Although many Traditional Chinese Medicine ingredients have strong pharmacological effects in vitro, their medicinal properties and clinical applications are seriously limited by many factors, such as poor water solubility, short half-life, poor stability, low bioavailability, high toxicity and side effects $[1,2,8]$.

Ly, an active alkaloid from a Traditional Chinese Medicine, Lycoris radiata (Amaryllidaceae), has received much attention as a promising anticancer agent [9-11]. We have found that Ly significantly induces the apoptotic and autophagic capacities of hepatocellular carcinoma (HCC) cells in vitro and in vivo. We also elucidate a novel molecular mechanism whereby Ly promotes apoptosis and autophagy through TCRP1/Akt/mTOR pathway in HCC [9]. Furthermore, we have identified Ly is an effective inhibitor of STAT3, leading to repression of multiple oncogenic processes in colon carcinoma [10]. Sun et.al reported that Ly possesses notable anticancer potentials in on-small cell lung carcinoma cells via blocking Wnt/ $\beta$ catenin signaling [12]. Hu et al. also demonstrated that Ly inhibits colorectal cancer development by targeting MEK2, involving autophagy-associated apoptosis [13]. Ly not only inhibits tumor growth, but also inhibits tumor metastasis. It has been reported that Ly can reduce the expression level of EMT related proteins. However, these results are limited to in vitro experiments and subcutaneously transplanted tumors. The effects and defined molecular mechanisms of Ly in inhibiting tumor growth and anti-metastasis in orthotopic transplanted tumor model, especially spontaneous tumor model, have 
It is of great significance to solve the problems of low solubility, poor stability, non-organizational specificity and severe side effects of Traditional Chinese Medicine [14]. Like many Traditional Chinese Medicine ingredients, the application of Ly is limited because of its poor solubility and low bioavailability. Research on improving the above issues of Ly by nanotechnology has not been reported. Recently, the carrier-free nanoparticles have emerged as an outstanding drug delivery system $[15,16]$. Moreover, unlike most intelligent nanocarriers which usually involve complex fabrication processes, such as materials synthesis, separation, purification, and modification, preparation of carrier-free nanoparticles by antisolvent method is simple and avoids batch-to-batch differentiation and quality challenges. Carrier-free nanomedicine could provide perfect drug loading and avoid undesired side effects from nonactive excipients or materials [17]. It is also helpful to minimize the interference of carrier materials to study the effects of nanoparticles on the key proteins of apoptosis [18], autophagy [19] and EMT [20].

Herein, we prepared carrier-free Ly nanoparticles (Ly NPs) and DS-Ly NPs by an anti-solvent method. Molecular validation mechanistically demonstrated that Ly and DS-Ly NPs induced apoptosis and autophagy in hepatocellular carcinoma (HepG2) cells and colonic carcinoma (SW480) in vitro. Ly and DSLy NPs also decreased proliferation, migration, invasion and EMT of these cancer cell lines (Fig. 1). Orthotopic xenotransplantation by implantation of $\mathrm{H} 22$ and spontaneous colonic tumor model were used to confirm in vivo anticancer effects and molecular mechanisms of Ly and DS-Ly NPs. Ly and DS-Ly NPs inhibited both growth and metastasis through many key proteins of apoptosis, autophagy and EMT. Enhanced antitumor effects of DS-Ly NPs were seen both in vitro and in vivo. In summary, such therapeutic carrier-free nanoparticle provides a new therapeutic strategy for treating cancer.

\section{Materials And Methods}

\subsection{Chemical and reagents}

Ly (purity $>98 \%$ ) was purchased from Yuanye Bio-Technology (Shanghai, China). 1,2-distearoyl-snglycero-3-phosphoethanolamine - $\mathrm{N}$ - [methoxy (polyethylene glycol)-3000] (DSPE-PEG) was purchased from Xiamen Sainuobangge Bio-Technology (xiamen, China).

\subsection{Preparation Ly NPs and DS-Ly NPs}

Ly NPs were preparation according to antisolvent precipitation method. The Ly powder was dissolved in ethanol. Briefly, $1 \mathrm{~mL}$ of Ly/ethanol solution was added into $10 \mathrm{~mL}$ ultrapure water and stirred for $30 \mathrm{~min}$ in a round-bottom flask in $60^{\circ} \mathrm{C}$ water baths. And then injected rapidly $2 \mathrm{~mL}$ DSPE-PEG solution $(0.4$ $\mathrm{mg} / \mathrm{mL}$ ) into Ly NPs for $1 \mathrm{~h}$.

\subsection{Characterization of Ly NPs and DS-Ly NPs}

The size distribution and surface charge of Ly NPs and DS-Ly NPs were measured by dynamic light scattering (DLS) at $25^{\circ} \mathrm{C}$. The Ly NPs and DS-Ly NPs diameter and morphology were evaluated by transmission electron microscope (TEM). 


\subsection{Drug loading}

The DS-Ly NPs were freeze-dried in the freeze dryer and were dissolved in the mixture of DMSO and water. The drug loading of DS-Ly NPs was determined and analyzed by the ultraviolet spectrophotometer method previously reported.

Loading content $(\%)=$ (amounts of Ly in nanoparticle) / (total amounts of Ly)

\subsection{Cell lines}

Human HCC cell lines HepG2, Human CRC cell lines, Human breast cancer cell lines were purchased from the Chinese Academy of Sciences (Beijing, China). The cells were cultured in high glucose Dulbecco's modified Eagle's medium (DMEM) supplemented with 10\% fetal bovine serum (FBS) and 1\% penicillin/streptomycin at $37^{\circ} \mathrm{C}$ in an incubator containing $5 \% \mathrm{CO} 2$ humidified atmosphere.

\subsection{Cell proliferation assays}

HepG2, SW480 and MCF-7 cells were seeded on a 96-well plate at density of 8000 cell/well and were treated with various concentrations $(0,2,4,6,8,10 \mu \mathrm{M})$ of Ly and DS-Ly NPs for $48 \mathrm{~h}$. The MTT solution were added and cultured for another $4 \mathrm{~h}$, and then the medium was discarded. $100 \mu \mathrm{L}$ DMSO was added and determined by microplate reader at the wavelength of $550 \mathrm{~nm}$.

\subsection{Colony formation assays}

Tumor cells were added into 6-well plates at a density of 1000 cells per well and cultured 7 days in incubator. Cells were treated with Ly and DS-Ly NPs at the same concentrations for $48 \mathrm{~h}$ and then continue culturing for 5 days. Next, we fixed the cells using $4 \%$ paraformaldehyde and then stained them with $0.1 \%$ crystal violet. The number of cells colonies was counted in indicated time periods.

\subsection{Western blot assays}

Western blot analysis was performed as previous described [21]. Antibodies against p62, Bax, Bcl-2, Vimentin and Cleaved caspase-3 were purchased from Cell Signaling Technology. Antibodies against LC3 , and E-cadherin were purchased from Proteintech. $\beta$-Actin antibodies were purchased from ABClonal. Full scans of western blot assays are shown in supplementary Fig. 2-5.

\subsection{Transwell migration and invasion assays}

In the migration and invasion assays, the transwell upper chambers were pre-coated with $50 \mu \mathrm{L}$ Matrigel for invasion and without Matrigel for migration. The cells were digested with trypsin, washed with PBS for 2-3 times, and the supernatant was centrifuged. The cells were resuspended to $1 \times 10^{5}$ cells $/ \mathrm{mL}$ in serum-free medium. $200 \mu \mathrm{L}$ suspension of HepG2 cells, SW480 cells and MCF-7 cells were respectively seeded into transwell upper chamber, and $600 \mu \mathrm{L}$ medium containing $10 \% \mathrm{FBS}$ and $1 \%$ penicillin/streptomycin was added in the lower chamber. After 24 hours of incubation in a constant 
temperature incubator with $5 \% \mathrm{CO}_{2}$ at $37^{\circ} \mathrm{C}$, the upper chamber medium was discarded, Ly and DS-Ly NPs were added respectively for 48 hours.

\subsection{Wound healing assays}

Tumor cells were seed into 6-well plates. When the tumor cells reached $80 \%-90 \%$ confluence, a sterile 200 $\mu \mathrm{L}$ tips was used to make a wound. To remove culture with cell debris and then filled with serum-free fresh medium containing drugs. After 48 hours, the percentages of migration cells were observed. Wound closure was recorded for each well by microscope and analyzed by Image $\mathrm{J}$ software.

\subsection{Animal experiments}

\subsubsection{AOM/DSS induced colorectal cancer}

The animals were adaptively fed for two weeks and then randomly divided into four groups: control (Con) group, model (Mod) group, Ly group and DS-Ly NPs group. Except for the control group, the other three groups were injected with $10 \mathrm{mg} / \mathrm{kg}$ azoxymethane (AOM) subcutaneously. Five days after AOM injection, the mice were given the first cycle of $2 \%$ dextran sulfate sodium (DSS)(w/v) in the drinking water for 5 days. After 5 consecutive days, the mice were fed with normal water for 2 weeks, and then perform a new cycle of $2 \%$ DSS feeding for 5 days and followed by another 2 weeks normal drinking water. The new cycle administration was repeated again. The mice were then intravenously administered Ly and DS-Ly NPs ( $5 \mathrm{mg} / \mathrm{kg}$ body weight) every two days. During the period, all the mice were observed in the body weight and were sacrificed at 12 th week.

\subsubsection{Orthotopic liver cancer model}

The Kunming mice were adaptively fed for one week, $\mathrm{H} 22$ cells $\left(5 \times 10^{6} / \mathrm{mL}\right)$ were injected subcutaneously into mice to build in-situ model. Subsequently, remove it from the skin when the subcutaneous tumor growth to $1 \mathrm{~cm}^{3}$. The tumor tissue is placed in saline and then cut into $1 \mathrm{~mm}^{3}$ pieces. The mice were anesthetized by intraperitoneal injection of Avertin and the skin was cut, the liver was squeezed out to expose the left lobe of the liver, about $3 \mathrm{~mm}$ incision was made in the left lobe of the liver, the tumor was implanted, and the abdominal cavity was sutured. The mice were then intravenously administered Ly and DS-Ly NPs ( $5 \mathrm{mg} / \mathrm{kg}$ body weight) every two days. During the period, all the mice were observed in the body weight and were sacrificed at 12 th week.

\subsection{Pharmacokinetic study}

To further study the concentration Ly and DS-Ly NPs in plasma samples, LC-MS/MS analysis were adopted. The rats were injected with Ly and DS-Ly NPs which were dissolved into Stroke-physiological saline solution via the intravenous injection at a dose of $5 \mathrm{mg} / \mathrm{kg}$. After $0.2 \mathrm{~mL}$ blood sample were collected into $1.5 \mathrm{~mL}$ tube containing heparin at $0.83,0.16,0.25,0.5,0.75,1,2,4,6,12,24 \mathrm{~h}$. The blood sample were centrifuged at $3000 \mathrm{rpm}$ for $15 \mathrm{~min}$. All sample were stored at $-80^{\circ} \mathrm{C}$ until used.

\subsection{Serum biochemical analysis}


Approximately $0.2 \mathrm{~mL}$ blood sample were collected into $1.5 \mathrm{~mL}$ tube. The blood sample were centrifuged at $3000 \mathrm{rpm}$ for $15 \mathrm{~min}$ and Serum was collected from the top fraction in another $1.5 \mathrm{~mL}$ tube. According to determine alanine aminotransferase (ALT) and aspartate transaminase (AST) levels.

\subsection{Statistical analysis}

All data are presented as mean \pm SD and were analyzed by prism software during the experiment. The

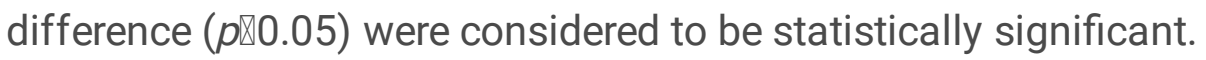

\section{Result}

\subsection{Construction and Characterization of Ly and DS-Ly NPs.}

DSPE-PEG-coated Ly NPs were prepared in two steps: Firstly, Ly NPs were prepared by a simple antisolvent precipitation method and kept at a certain temperature (Fig. 2a). Subsequently, DSPE-PEG was added to Ly NPs suspension to achieve long blood circulation and water-soluble stability. Ly NPs showed a hydrodynamic diameter of $43.18 \pm 0.16 \mathrm{~nm}$ (polydispersity index, PDI $=0.233$ ) as examined by dynamic light scattering (DLS) measurement. PEG-modified Ly NPs (DS-Ly NPs) remained monodisperse (PDI around 0.172 ), with their hydrodynamic sizes rising to $48.48 \pm 0.50 \mathrm{~nm}$ as a result of PEGylation (Fig. $2 \mathrm{~b}$ ). TEM further revealed uniformed particles size and spherical morphology of DS-Ly NPs (Fig. 2C).

The particle size of Ly NPs modified with DSPE-PEG remained relatively stable within 7 days, while the size of the nanoparticles without DSPE-PEG was obviously increased (Fig. 2d). In addition, zeta potential is also an important indicator to detect stability of nano-formulation. PDI represents the size distribution width of particles and the value less than 0.5 indicates good distribution. The zeta potential and PDI of the DS-Ly NPs kept relatively stable within 7 days (Fig. 2e). Therefore, these results showed that DSPEPEG can also increase nanoparticle stability. The drug loading of coated DSPE-PEG based on Ly NPS were $73.92 \pm 7.33 \%$. Taken together, nanoparticles with good stability and high drug loading have been prepared.

\subsection{DS-Ly NPs enhanced the toxicity and apoptosis- promoting of Ly to tumor.}

The viability and clonogenicity ability of DS-Ly NPs were evaluated using human hepatoma carcinoma cell line (HepG2), human breast cancer cell line (MCF-7) and human colorectal cancer cell line (SW480), respectively. The viability and colony formation ability of DS-Ly NPs against three kinds of human cancer cells, specifically HepG2, SW480 and MCF-7 cells, were evaluated by using MTT and crystal violet staining. DS-Ly NPs can significantly reduce viability and clonogenicity compared with Ly towards HepG2, SW480 and MCF-7 cells (Fig. 3a-b). Furthermore, to demonstrate that Ly, effectively released from DS-Ly NPs, could also induce apoptosis, the western blot assays were performed to compare the apoptosis-inducing capabilities of Ly and DS-Ly NPs. The Bax and Cleaved caspase-3 protein levels in 
and upregulation was improved by DS-Ly NPs. Instead, compared with the Con group, the Bcl-2 protein levels in HepG2, SW480 and MCF-7 cells treated with DS-Ly NPs were lower than that in tumor cells treated with Ly alone (Fig. 3c). Meanwhile, the ratio of Bax/Bcl-2 expression increased significantly after DS-Ly NPs treatment compared with Ly (Fig. 3d). Taken together, these results indicate that DS-Ly NPs could enhance the toxicity and apoptosis induced by Ly.

\subsection{DS-Ly NPs improved the promotion of autophagy and inhibition of migration and invasion of Ly to tumor.}

To study the effect of Ly and DS-Ly NPs on HepG2, MCF-7, SW480 cells migration and invasion, respectively; we performed wound healing assays and transwell assays to evaluate the effect on cancer cell metastasis. In the transwell assays, the number of invaded cells has decreased markedly after DS-Ly NPs compared with Ly (Fig. 4a-b). Wound healing assays demonstrated that there was a markedly increase in wound closure distance of DS-Ly NPs in comparison with Ly (Fig. 4C). This was further confirmed by western blot. As indicated in Fig. 4d, compared to Con, DS-Ly NPs significantly increased the expression of E-cadherin protein, while markedly reduced the expression of Vimentin protein. These results demonstrate that DS-Ly NPs exhibits a better ability to inhibit cancer cell metastasis than Ly.

Previous studies in our lab have reported that Ly can induce autophagy; we next detected the ability of Ly and DS-Ly NPs to induce autophagy in cancer cells. The amounts of LC-3 were significantly increased and p62 was markedly decreased by treatment with DS-Ly NPs than that by the same concentration of Ly (Fig. 4e). Apparently, the autophagy effect of DS-Ly NPs towards cancer cells was markedly increased than that of Ly.

\subsection{The inhibitory effect of DS-Ly NPs on liver cancer is more obvious than that of Ly in vivo.}

To further investigate the DS-Ly NPs anticancer efficacy, we established an orthotopic liver cancer model. As shown in Fig. 5a-b, Ly and DS-Ly NPs had remarkable effects on suppressing tumor growth compared with saline as a negative control after intravenous injection into the tumor-bearing mice. DS-Ly NPs presented remarkably higher therapeutic efficacy towards tumor growth than Ly, which mainly due to the tumor targeting capability of DS-Ly NPs (Fig. 5d-e). Furthermore, Histological Examination (HE) indicated that Ly and DS-Ly NPs suppressed the lung metastasis, and DS-Ly NPs was significantly reduced as compared to Ly (Fig. 5c). The weight of mice did not significantly change during the administration (Fig. 5f). To further determine whether tumor can happen apoptosis, autography and metastasis in vivo, we utilized western blot analysis to detect the change of their marker. These results reveal that DS-Ly NPS can better upregulate expression of Cleaved caspase-3, Bax, LC-3 and E-cadherin and down-regulate the expression of p62 compared with Ly. (Fig. 5g). Meanwhile, Immunohistochemistry (IHC) has also verified that DS-Ly NPs was markedly increased the expression of E-cadherin and LC-3 and reduced the expression of Ki67 compared with Ly (Fig. 5h-i). Taken together, these results show that DS-Ly NPs could improve the HCC inhibition effect of Ly against cancer cells in vivo. 
Furthermore, for better verification, we explored the role of Ly and DS-Ly NPs in colorectal cancer. We established the AOM/DSS model in C57BL/ 6 male mice, following by Tail vein injection with Ly, DS-Ly NPs or the saline (Fig. 6a). We observed that DS-Ly NPs treatment also protected C57BL/6 male mice from the AOM/DSS induced colorectal cancer. Compared to Ly, DS-Ly NPs treatment obviously extended colon lengths (Fig. $6 \mathrm{~b}$ and d), and reduced the volume and number of tumors (Fig. 6c). In addition, during the experiment, we focused on the body weight of the mice, which proved that the weight of the mice in the DS-Ly NPs group recovered obviously than other groups except normal groups (Fig. 6f). HE results showed that glands of the normal group were well-arranged and contained abundant goblet cells (Fig. 6e). However, the model group had more disordered glandular arrangement and fewer goblet cells than the normal group. After administration of the DS-Ly NPs, the pathological state was improved, the glands tended to be arranged regularly, and the number of goblet cells increased than Ly. Furthermore, compared to Ly, DS-Ly NPs further upregulated Cleaved caspase-3, E-cadherin and LC-3 and downregulated vimentin and p62 by western blot assays (Fig. $6 \mathrm{~h}$ ).

We next explored the expression of E-cadherin, LC-3 and Ki67 by Immunohistochemistry. The results showed that the expression of E-cadherin and LC-3 markedly increased after Ly and DS-Ly NPs treatment, however, DS-Ly NPs treatment showed greater potencies for upregulating the protein expression than Ly treatment. It is suggested that Ly and DS-Ly NPs could suppress metastasis and promote autography of colon cancer, while DS-Ly NPs has better therapeutic effect. Meanwhile, the expression of Ki67 significantly decreased compared with Ly (Fig. $6 \mathrm{~g}$ and i). Taken together, the anti-tumor effect becomes apparent by DS-Ly NPs compared with Ly in vivo.

\subsection{Pharmacokinetics study of Ly and DS-Ly NPs in vivo.}

The concentration of Ly in rat plasma was measured by LC-MS/MS method. The mean plasma concentration of Ly in serum after tail vein injection of Ly and DS-Ly NPs $(5 \mathrm{mg} / \mathrm{kg})$ in healthy rats. The mean plasma concentration curve and parameters as is shown in supplementary Fig. 1 and supplementary Table 1. The half-time of Ly and DS-Ly NPs were $1.068 \pm 0.205 \mathrm{~h}$ and $2.13 \pm 1.058 \mathrm{~h}$. These results shown that DS-Ly NPs can better prolonged and the utilization rate was improved than Ly.

\subsection{Evaluation of AST and ALT levels of Ly and DS-Ly NPs in vivo.}

To further study the toxicity of Ly and DS-Ly NPs, ALT and AST of rat serum were determined by biochemical analyzer. The result shows that there no significance difference of serum AST and ALT among control, DS-Ly NPS. However, the ALT and AST ratio of Ly were improved significantly. These results display that DS-Ly NPs has reduced the toxicity compared with Ly.

In this study, we successfully prepared a carrier-free nanoparticle based on DSPE-PEG modification with satisfying morphological characterization and stability. The above research results show that DS-Ly NPS displays a superior anti-tumor effect against liver cancer, colorectal cancer and breast cancer compared to Lv accordina to cell viabilitv. apoptosis. autophaav and metastasis in vitro and in vivo. Meanwhile, DSLoading [MathJax]/jax/output/CommonHTML/fonts/TeX/fontdata.js 
Ly NPs improved the half-life, extend the time and reduce the toxicity compared to Ly in vivo. This research demonstrated that DS-Ly NPs could be a promising therapeutic drug for tumor treatment in future.

\section{Discussion}

Natural products play a very important role in the prevention and treatment of diseases [22, 23]. Ly, an active natural product, has been reported to have a wide range of anti-tumor activities [24, 25]. However, due to its short half-life and low water solubility, its absorption and application in the body have been greatly restricted [26]. In this study, we prepared a DS-Ly NPs to overcome these drawbacks. At the same time, our studies have found that modified Ly may further enhance its anti-tumor effect in vitro and in vivo.

With the development of nanoscience and nanotechnology, nano-preparations such as carrier-free nanoparticles, micelles, nanoliposomes, and nanogels have been used to treat various malignant tumors [27]. Guo et al. found that mannosylation can inhibit tumor cell proliferation and enhance tumor cell uptake through selective targeted delivery of Ly [26]. Liu et al. showed that the FITC-labeled MSN/PEG/Lycorine/Antibody, as potential drug carrier, can effectively release drug and suppress growth of prostate cancer cells [28]. It is worth noting that the carrier-free nanoparticles promote Ly absorption and reduce its toxic side effects. At the same time, Ly nanoparticles exhibit good anti-tumor activity in vitro, but they have not been reported yet in vivo.

Previous studies reported that the DSPE-PEG modified nanocarrier system can significantly extend the circulation time of nanoparticles in the blood $[29,30]$. In this study, we used a simple solvent exchange method to prepare carrier-free Ly NPs by regulating the supersaturation of hydrophobic drugs in water and modifying with DSPE-PEG. DS-Ly NPs is completely composed of Ly and DSPE-PEG, without the participation of inactive excipients or materials, which can provide suitable drug loading and avoid the adverse side effects caused by inactive excipients or materials [31-33]. At the same time, the biological safety and metabolic problems that may be caused by the use of a large number of inert carriers in traditional drug delivery systems can be avoided in vivo [34,35]. In addition, our studies have found that Ly NPs have an effect on cell apoptosis, autophagy and EMT, which also helps to minimize the interference of carrier materials.

In our previous research, we found that Ly has a wide range of anti-tumor activities including apoptosis, autophagy, proliferation and so on [36-38]. In this study, we intend to compare whether DS-Ly NPs has better anti-tumor effects than Ly. We found that DS-Ly NPs can significantly reduce viability and clonogenicity compared with Ly towards HepG2, SW480 and MCF-7 cells. We also observed the protein levels of Cleaved caspase-3 and the ratio of Bax/Bcl-2 were dramatically higher in DS-Ly NPs than in Ly. Taken together, our results suggested that DS-Ly NPs showed better growth-suppressive activity and promotion of apoptosis than Ly in three cancer cells. Moreover, we also compared the effects of Ly on tumnr invacinn and motactacic hof nro and aftor narriar-free nano-modification. 
EMT has attracted more and more attention in promoting tumor cell invasion and metastasis [39-41]. In this study, we also determined the protein levels of two specific EMT markers, E-cadherin and Vimentin. We found that DS-Ly NPs treatment led to a significantly increased expression of E-cadherin, while decreased expression of Vimentin compared with Ly. To further compare the effects of Ly before and after modification on tumor invasion and migration, we performed wound healing assays and transwell assays. These results showed that the number of invaded cells was decreased markedly and wound closure distance was dramatically increased after DS-Ly NPs compared with Ly. Collectively, our results suggest that DS-Ly NPs significantly suppress invasion and metastasis compared with Ly in cancer cells.

Autophagy is another way of programmed cell death. In our previous study [42-44], we have demonstrated that Ly induces autophagy in HCC cells. In this study, we compared the effects of Ly on tumor autophagy before and after carrier-free nano-modification. We found that DS-Ly NPs noticeably increased the expression of LC-3, while reduced the expression of p62. Collectively, our results indicate that DS-Ly NPs could markedly promote autophagy compared with Ly in cancer cells.

Although previous reports have already demonstrated that Ly possessed obvious anticancer effect in vivo, its nanoformulation had not yet been fully proved. According to previous reports, Ly has been found to have a preferable effect on subcutaneous tumors in mouse, but the role of Ly on orthotopic Hepatocellular carcinoma and spontaneous colorectal cancer model in mice has not been investigated. In this study, we established an orthotopic mouse model of liver cancer to detect the effect of Ly and DS-Ly NPs on tumor tissue. We found that Ly and DS-Ly NPs had remarkable effects on suppressing tumor growth compared with saline as a negative control after intravenous injection into the tumor-bearing mice. DS-Ly NPs presented remarkably higher therapeutic efficacy towards tumor growth than Ly, which mainly due to the tumor targeting capability and effective blood circulation of DS-Ly NPs. Furthermore, HE indicated that DS-Ly NPs suppressed the lung metastatic, which DS-Ly NPs was significantly reduced as compared to control and Ly group. In addition, our results revealed that compared with Ly, DS-Ly NPS can dramatically upregulate expression of Cleaved caspase-3, Bax, LC-3 and E-cadherin, and downregulate expression of p62 and Ki67 by western blot assays and Immunohistochemistry assays. Moreover, we also established the AOM/DSS-induced mouse colorectal cancer to detect the effect of Ly and DS-Ly NPs on tumor tissue. Compared to Ly, DS-Ly NPs treatment obviously extended colon length, and reduced the volume and number of tumors. In addition, during the experiment, we focused on the body weight of the mice, which proved that the weight of the mice in the DS-Ly NPs group recovered obviously than other groups except normal groups. HE staining results showed that after administration of the DS-Ly NPs, the pathological state was improved, the glands tended to be arranged regularly, and the number of goblet cells increased compared with control and Ly group. Furthermore, we found that DSLy NPs further upregulated Cleaved caspase-3, E-cadherin and LC-3 and downregulated Vimentin and p62 compared with Ly group by western blot assays. We also explored the expression of E-cadherin, LC-3 and Ki67 by Immunohistochemistry assays. The results showed that the expression of E-cadherin and LC-3 markedly increased after DS-Ly NPs treatment compared with control and Ly group. Taken together, our results suggest that DS-Ly NPs dramatically inhibits tumor growth compared with Ly by inducing 
autography and apoptosis and suppresses cancer metastasis to inhibit the occurrence and development of cancer in vivo.

\section{Conclusion}

In this study, we prepared a DS-Ly NPs. We find that compared with Ly, DS-Ly NPs can significantly inhibit cell viability. Meanwhile, DS-Ly NPs treatment results in higher promotion of apoptotic and autophagic capacities, as well as inhibition of migration and invasion. Furthermore, DS-Ly NPs exhibits a better antitumor effect in AOM/DSS induced colorectal tumors and orthotopic hepatocellular tumors than Ly in repression of multiple oncogenic processes, which may benefit from the advantages of carrier-free nanoparticle. Taken together, these results have demonstrated that carrier-free nanoparticle provides a new therapeutic strategy for treating cancer.

\section{Abbreviations}

HCC, hepatocellular carcinoma; DMSO, dimethyl sulfoxide; DMEM, Dulbecco's modified Eagle's medium; FBS, fetal bovine serum; TEM, transmission electron microscopy; IHC, immunohistochemistry; H\&E, hematoxylin and eosin;

\section{Declarations}

\section{Acknowledgements}

We thank Mr. Hongwei Liu and Kailong Wang (Tianjin University of Traditional Chinese Medicine) for his technical assistance in animal experiments.

\section{Financial support}

This work was supported by grants from National Natural Science Foundation of China (Nos. 81873089 and 81603253 to H. Yu, No. 81501578 to B. Du, No. 81973570 to Y. Qiu), Important Drug Development Fund, Ministry of Science and Technology of China (No. 2018ZX092011 to Z. Li), Science and Technology of Tianjin (No. 20ZYJDJC00070 to Y. Wang).

\section{Conflict of interest}

The authors disclose no potential conflicts of interest.

\section{Author contributions}

H. Yu and B. Du contributed to conception and design. S. Zhou, R. Liu, S. Yin, Y. Shao, S. Wu performed experiments. S. Zhou, Y. Qiu, Q. Chen, T. Wang, B. Du, H. Yu analyzed data. S. Zhou, Y. Qiu, B. Du, H. Yu wrote the manuscript. 


\section{Ethics approval and consent to participate}

Animal studies were conducted according to protocols approved by Guidance Suggestions for the Care and Use of Laboratory Animals issued by the Ministry of Science and Technology of China (2006), and the Animal Use and Care Committee of Tianjin University of Traditional Chinese Medicine.

\section{Consent for publication}

All authors concur with the submission and publication of this paper.

\section{Availability of data and materials}

All data generated or analyzed during this study are included in this published article and the Additional Information.

\section{References}

1. Zhang J, Li X, Huang L: Anticancer activities of phytoconstituents and their liposomal targeting strategies against tumor cells and the microenvironment. Adv Drug Deliv Rev 2020, 154-155:245-273.

2. Feng X, Cao S, Qiu F, Zhang B: Traditional application and modern pharmacological research of Artemisia annua L. Pharmacol Ther 2020, 216:107650.

3. Akin EJ, Alsaloum M, Higerd GP, Liu S, Zhao P, Dib-Hajj FB, Waxman SG, Dib-Hajj SD: Paclitaxel increases axonal localization and vesicular trafficking of Nav1.7. Brain 2021.

4. Yin S, Qiu Y, Jin C, Wang R, Wu S, Liu H, Koo S, Han L, Zhang Y, Gao X, et al: 7-Deoxynarciclasine shows promising antitumor efficacy by targeting Akt against hepatocellular carcinoma. Int J Cancer 2019, 145:3334-3346.

5. Wang H, Yang S, Zhou H, Sun M, Du L, Wei M, Luo M, Huang J, Deng H, Feng Y, et al: Aloperine executes antitumor effects against multiple myeloma through dual apoptotic mechanisms. J Hematol Oncol 2015, 8:26.

6. Sun D, Tao W, Zhang F, Shen W, Tan J, Li L, Meng Q, Chen Y, Yang Y, Cheng H: Trifolirhizin induces autophagy-dependent apoptosis in colon cancer via AMPK/mTOR signaling. Signal Transduct Target Ther 2020, 5:174.

7. Wang D, Wang Y, Zhao G, Zhuang J, Wu W: Improving systemic circulation of paclitaxel nanocrystals by surface hybridization of DSPE-PEG2000. Colloids Surf B Biointerfaces 2019, 182:110337.

8. Hu J, Dong Y, Ding L, Dong Y, Wu Z, Wang W, Shen M, Duan Y: Local delivery of arsenic trioxide nanoparticles for hepatocellular carcinoma treatment. Signal Transduct Target Ther 2019, 4:28. 
9. Yu H, Qiu Y, Pang X, Li J, Wu S, Yin S, Han L, Zhang Y, Jin C, Gao X, et al: Lycorine Promotes Autophagy and Apoptosis via TCRP1/Akt/mTOR Axis Inactivation in Human Hepatocellular Carcinoma. Mol Cancer Ther 2017, 16:2711-2723.

10. Wu S, Qiu Y, Shao Y, Yin S, Wang R, Pang X, Ma J, Zhang C, Wu B, Koo S, et al: Lycorine Displays Potent Antitumor Efficacy in Colon Carcinoma by Targeting STAT3. Front Pharmacol 2018, 9:881.

11. Roy M, Liang L, Xiao X, Feng P, Ye M, Liu J: Lycorine: A prospective natural lead for anticancer drug discovery. Biomed Pharmacother 2018, 107:615-624.

12. Sun Y, Wu P, Sun Y, Sharopov FS, Yang Q, Chen F, Wang P, Liang Z: Lycorine possesses notable anticancer potentials in on-small cell lung carcinoma cells via blocking Wnt/beta-catenin signaling and epithelial-mesenchymal transition (EMT). Biochem Biophys Res Commun 2018, 495:911-921.

13. Hu M, Yu Z, Mei P, Li J, Luo D, Zhang H, Zhou M, Liang F, Chen R: Lycorine Induces autophagyassociated apoptosis by targeting MEK2. Aging 2020, 12:1.

14. Liu J, Wang Y, Qiu Z, Lv G, Huang X, Lin H, Lin Z, Qu P: Impact of TCM on Tumor-Infiltrating Myeloid Precursors in the Tumor Microenvironment. Front Cell Dev Biol 2021, 9:635122.

15. Wang C, Li L, Zhang S, Yan Y, Huang Q, Cai X, Xiao J, Cheng Y: Carrier-Free Platinum Nanomedicine for Targeted Cancer Therapy. Small 2020, 16:e2004829.

16. Zhu L, Guo Y, Qian Q, Yan D, Li Y, Zhu X, Zhang C: Carrier-Free Delivery of Precise Drug-Chemogene Conjugates for Synergistic Treatment of Drug-Resistant Cancer. Angew Chem Int Ed Engl 2020, 59:1794417950.

17. Zhang N, Li M, Sun X, Jia H, Liu W: NIR-responsive cancer cytomembrane-cloaked carrier-free nanosystems for highly efficient and self-targeted tumor drug delivery. Biomaterials 2018, 159:25-36.

18. Supper E, Rudat S, lyer V, Droop A, Wong K, Spinella JF, Thomas P, Sauvageau G, Adams DJ, Wong CC: Cut-like homeobox 1 (CUX1) tumor suppressor gene haploinsufficiency induces apoptosis evasion to sustain myeloid leukemia. Nat Commun 2021, 12:2482.

19. Ligeon LA, Pena-Francesch M, Vanoaica LD, Nunez NG, Talwar D, Dick TP, Munz C: Oxidation inhibits autophagy protein deconjugation from phagosomes to sustain $\mathrm{MHC}$ class II restricted antigen presentation. Nat Commun 2021, 12:1508.

20. Klatt Shaw D, Saraswathy VM, Zhou L, McAdow AR, Burris B, Butka E, Morris SA, Dietmann S, Mokalled MH: Localized EMT reprograms glial progenitors to promote spinal cord repair. Dev Cell 2021, 56:613-626 e617.

21. Wu T, Wang C, Wang W, Hui Y, Zhang R, Qiao L, Dai Y: Embelin impairs the accumulation and Oncoimmunology 2018, 7:e1498437. 
22. Schafer M, Semmler ML, Bernhardt T, Fischer T, Kakkassery V, Ramer R, Hein M, Bekeschus S, Langer P, Hinz B, et al: Small Molecules in the Treatment of Squamous Cell Carcinomas: Focus on Indirubins. Cancers (Basel) 2021, 13.

23. Liang Q, Cai W, Zhao Y, Xu H, Tang H, Chen D, Qian F, Sun L: Lycorine ameliorates bleomycin-induced pulmonary fibrosis via inhibiting NLRP3 inflammasome activation and pyroptosis. Pharmacol Res 2020, 158:104884.

24. Li C, Deng C, Pan G, Wang X, Zhang K, Dong Z, Zhao G, Tan M, Hu X, Shi S, et al: Lycorine hydrochloride inhibits cell proliferation and induces apoptosis through promoting FBXW7-MCL1 axis in gastric cancer. J Exp Clin Cancer Res 2020, 39:230.

25. Zhang P, Zhang M, Yu D, Liu W, Hu L, Zhang B, Zhou Q, Cao Z: Lycorine inhibits melanoma cell migration and metastasis mainly through reducing intracellular levels of beta-catenin and matrix metallopeptidase 9. J Cell Physiol 2019, 234:10566-10575.

26. Guo Y, Liu X, Sun X, Zhang Q, Gong T, Zhang Z: Mannosylated lipid nano-emulsions loaded with lycorine-oleic acid ionic complex for tumor cell-specific delivery. Theranostics 2012, 2:1104-1114.

27. Wong KE, Ngai SC, Chan KG, Lee LH, Goh BH, Chuah LH: Curcumin Nanoformulations for Colorectal Cancer: A Review. Front Pharmacol 2019, 10:152.

28. Liu X, Kang J, Wang H, Huang T, Li C: Construction of Fluorescein Isothiocyanate-Labeled MSNs/PEG/Lycorine/Antibody as Drug Carrier for Targeting Prostate Cancer Cells. J Nanosci Nanotechnol 2018, 18:4471-4477.

29. Sun J, Li Y, Teng Y, Wang S, Guo J, Wang C: NIR-controlled HSP90 inhibitor release from hollow mesoporous nanocarbon for synergistic tumor photothermal therapy guided by photoacoustic imaging. Nanoscale 2020, 12:14775-14787.

30. Damasco JA, Chen G, Shao W, Agren H, Huang H, Song W, Lovell JF, Prasad PN: Size-tunable and monodisperse $\mathrm{Tm}(3)(+) / \mathrm{Gd}(3)(+)$-doped hexagonal $\mathrm{NaYbF}(4)$ nanoparticles with engineered efficient near infrared-to-near infrared upconversion for in vivo imaging. ACS Appl Mater Interfaces 2014, 6:1388413893.

31. Li Y, Lin J, Cai Z, Wang P, Luo Q, Yao C, Zhang Y, Hou Z, Liu J, Liu X: Tumor microenvironmentactivated self-recognizing nanodrug through directly tailored assembly of small-molecules for targeted synergistic chemotherapy. Journal of Controlled Release 2020, 321:222-235.

32. Li Y, Lin J, Wang P, Luo Q, Lin H, Zhang Y, Hou Z, Liu J, Liu X: Tumor Microenvironment Responsive Shape-Reversal Self-Targeting Virus-Inspired Nanodrug for Imaging-Guided Near-Infrared-II Photothermal Chemotherapy. ACS Nano 2019, 13:12912-12928. 
33. Li Y, Liu G, Ma J, Lin J, Lin H, Su G, Chen D, Ye S, Chen X, Zhu X, Hou Z: Chemotherapeutic drugphotothermal agent co-self-assembling nanoparticles for near-infrared fluorescence and photoacoustic dual-modal imaging-guided chemo-photothermal synergistic therapy. Journal of Controlled Release 2017, 258:95-107.

34. Wen Y, Zhang W, Gong N, Wang YF, Guo HB, Guo W, Wang PC, Liang XJ: Carrier-free, self-assembled pure drug nanorods composed of 10-hydroxycamptothecin and chlorin e6 for combinatorial chemophotodynamic antitumor therapy in vivo. Nanoscale 2017, 9:14347-14356.

35. Park J, Sun B, Yeo Y: Albumin-coated nanocrystals for carrier-free delivery of paclitaxel. J Control Release 2017, 263:90-101.

36. Zhao Z, Xiang S, Qi J, Wei Y, Zhang M, Yao J, Zhang T, Meng M, Wang X, Zhou Q: Correction of the tumor suppressor Salvador homolog-1 deficiency in tumors by lycorine as a new strategy in lung cancer therapy. Cell Death Dis 2020, 11:387.

37. Roy M, Liang L, Xiao X, Peng Y, Luo Y, Zhou W, Zhang J, Qiu L, Zhang S, Liu F, et al: Lycorine Downregulates HMGB1 to Inhibit Autophagy and Enhances Bortezomib Activity in Multiple Myeloma. Theranostics 2016, 6:2209-2224.

38. Hayden RE, Pratt G, Drayson MT, Bunce CM: Lycorine sensitizes CD40 ligand-protected chronic lymphocytic leukemia cells to bezafibrate- and medroxyprogesterone acetate-induced apoptosis but dasatanib does not overcome reported CD40-mediated drug resistance. Haematologica 2010, 95:18891896.

39. Maimon A, Levy-Yahid V, Ben-Meir2 K. Myeloid cell-derived PROS1 inhibits tumor metastasis by regulating inflammatory and immune responses via IL-10, J Clin Invest, 85 (2021) 1-40.

40. Zhang B, Li Y, Wu Q, Xie L, Barwick B, Fu C, Li X, Wu D, Xia S, Chen J, et al: Acetylation of KLF5 maintains EMT and tumorigenicity to cause chemoresistant bone metastasis in prostate cancer. Nat Commun 2021, 12:1714.

41. Yang S, Li H, Yao H, Zhang Y, Bao H, Wu L, Zhang C, Li M, Feng L, Zhang J, et al: Long noncoding RNA ERLR mediates epithelial-mesenchymal transition of retinal pigment epithelial cells and promotes experimental proliferative vitreoretinopathy. Cell Death Differ 2021.

42. Cui YH, Yang S, Wei J, Shea CR, Zhong W, Wang F, Shah P, Kibriya MG, Cui X, Ahsan H, et al: Autophagy of the m(6)A mRNA demethylase FTO is impaired by low-level arsenic exposure to promote tumorigenesis. Nat Commun 2021, 12:2183.

43. Rosenfeldt MT, O'Prey J, Lindsay CR, Nixon C, Roth S, Sansom OJ, Ryan KM: Loss of autophagy affects melanoma development in a manner dependent on PTEN status. Cell Death Differ 2021, 28:14371420 Loading [MathJax]/jax/output/CommonHTML/fonts/TeX/fontdata.js 
44. Lv Y, Zhang W, Zhao J, Sun B, Qi Y, Ji H, Chen C, Zhang J, Sheng J, Wang T, et al: SRSF1 inhibits autophagy through regulating $\mathrm{Bcl}-\mathrm{x}$ splicing and interacting with PIK3C3 in lung cancer. Signal Transduct Target Ther 2021, 6:108.

\section{Figures}

\section{Figure 1}

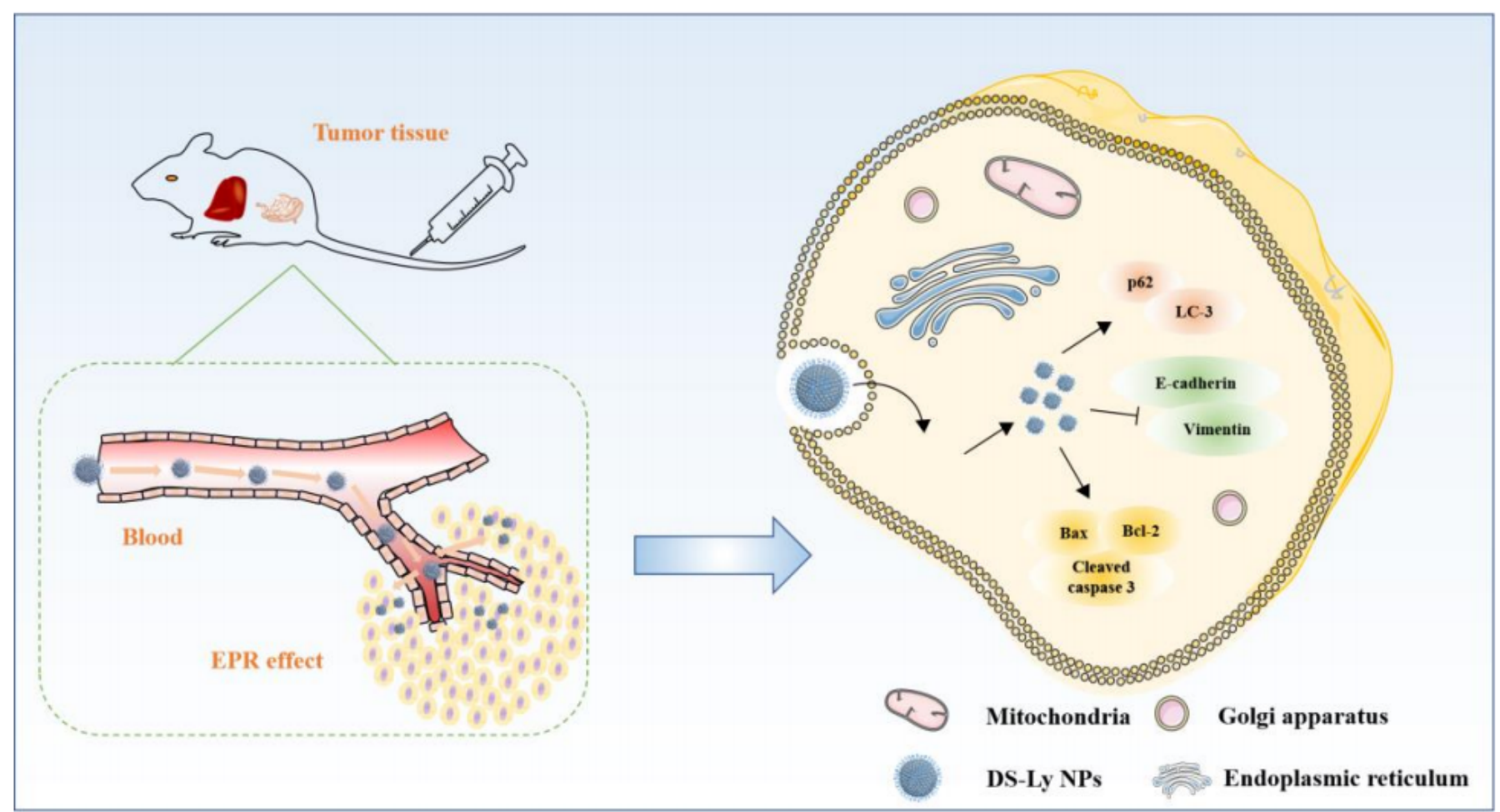

Figure 1

Schematic representation of the preparation of DS-Ly NPs through self-assemble for antitumor therapy. 
Figure 2

(a)

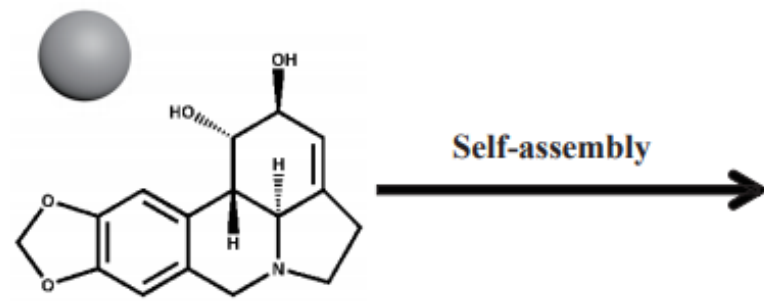

Ly

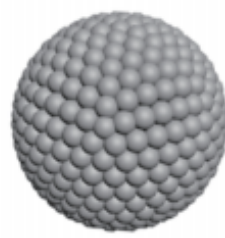

Ly NPs

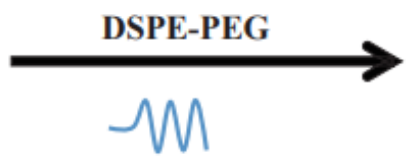

(c)

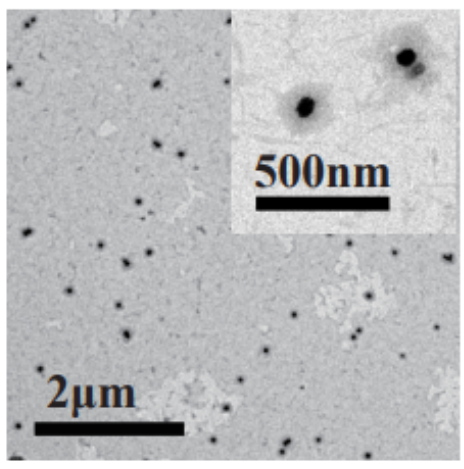

(e)

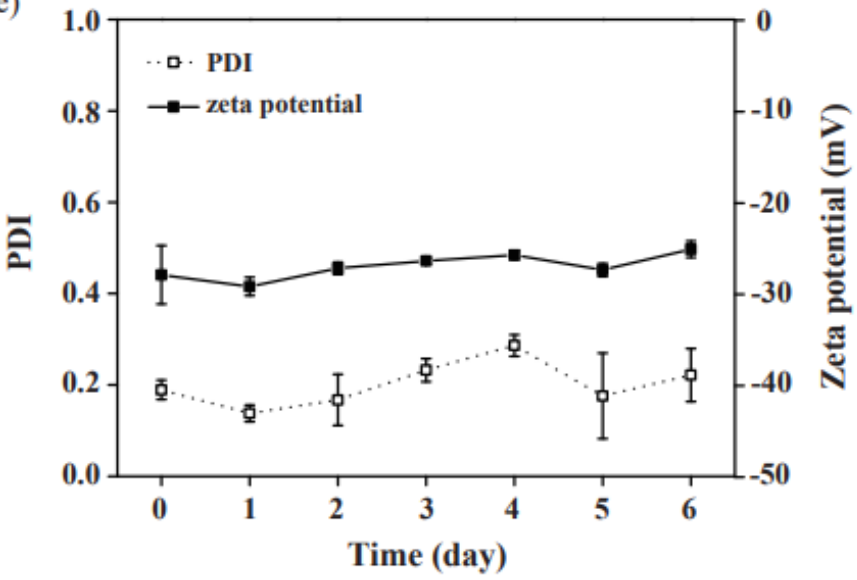

Figure 2

Characterization of Ly NPs and DS-Ly NPs. (a) Schematic description of Ly NPs and DS-Ly NPs preparation. (b) Size distribution of Ly NPs and DS-Ly NPs. (c) Transmission electron microscopy (TEM) image of Ly NPs and DS-Ly NPs. (d) Size stability of Ly NPs and DS-Ly NPs in 6 days. (e) PDI and zeta potential stability of Ly NPs and DS-Ly NPs in 6 days. 
Figure 3

(a)

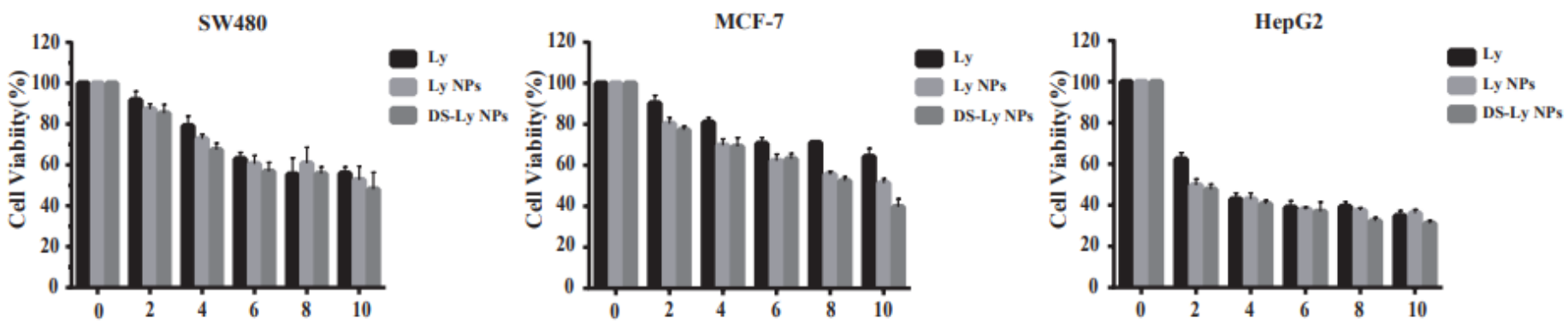

(b)
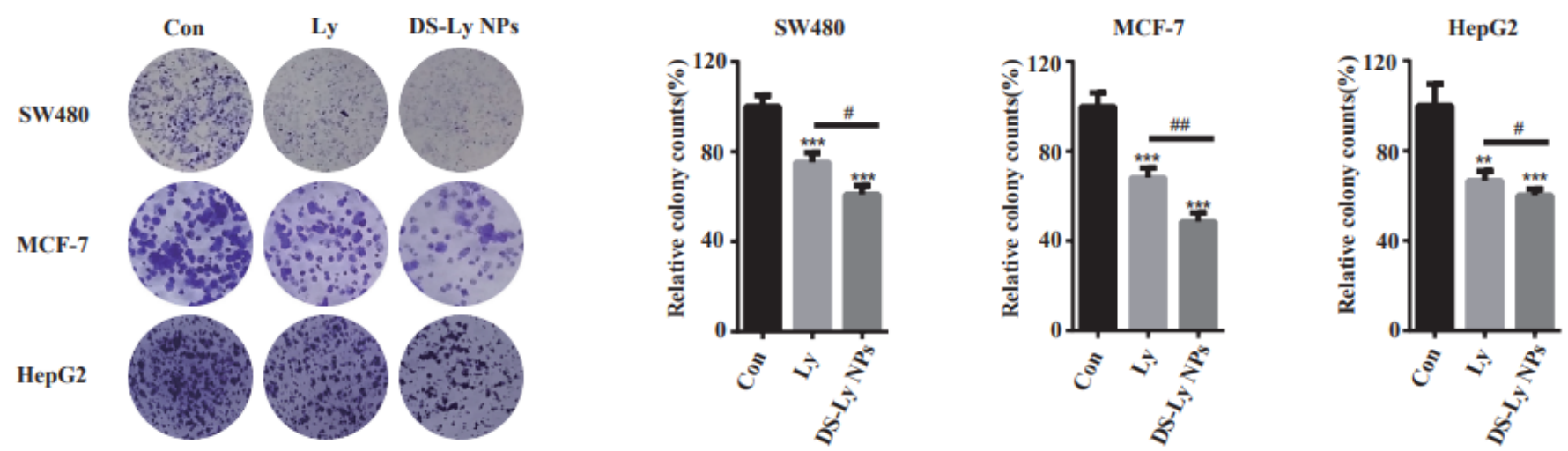

(c)
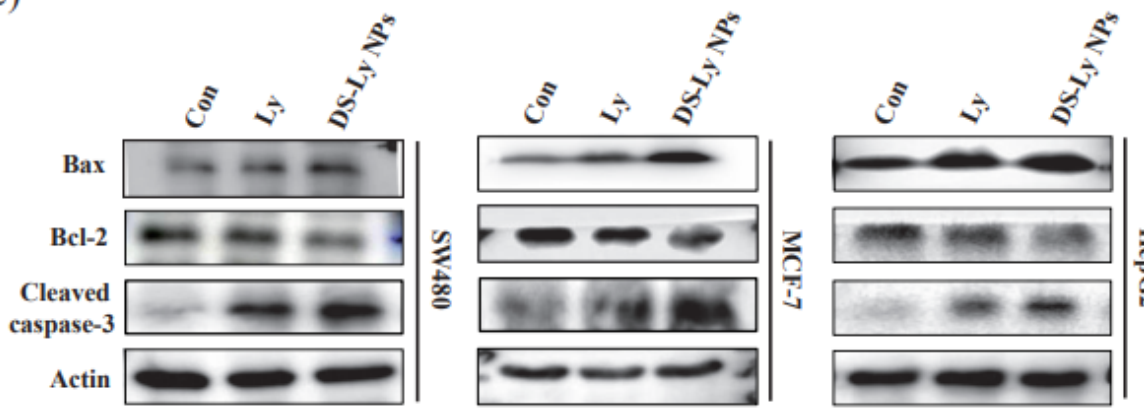

(d)
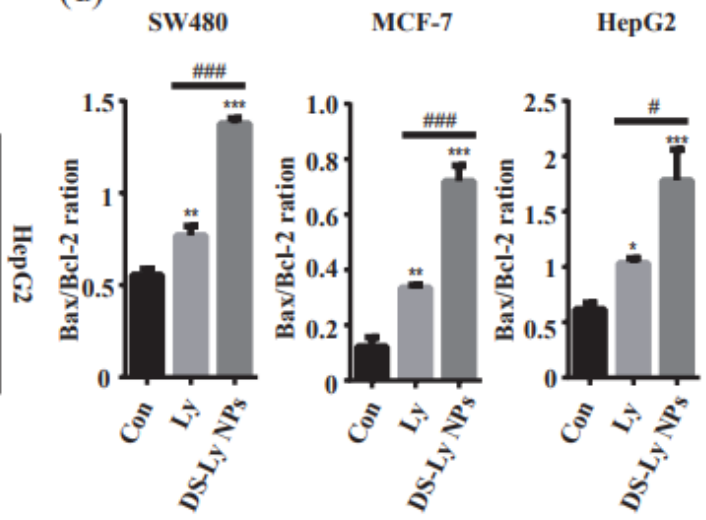

\section{Figure 3}

Ly and DS-Ly NPs inhibits cell proliferation and triggers cell apoptosis in SW480, MCF-7 and HepG2 cells.

(a) Cell viability was measured using the MTT assay. (b) The colongenicity of SW480, MCF-7 and HepG2 cells. (c) The protein levels of Cleaved caspase-3, Bax and Bcl-2 were determined by western blot assays.

(d) The ratio of Bax and Bcl-2. ${ }^{*} \mathrm{p}<0.05 ;{ }^{* *} \mathrm{p}<0.01$; ${ }^{* *} \mathrm{p}<0.001$ compared to control. \#p $<0.05$, \#\#p < 0.01 ,\#\# $<0.001$ compared to control treated with Ly (Student's t-test). 

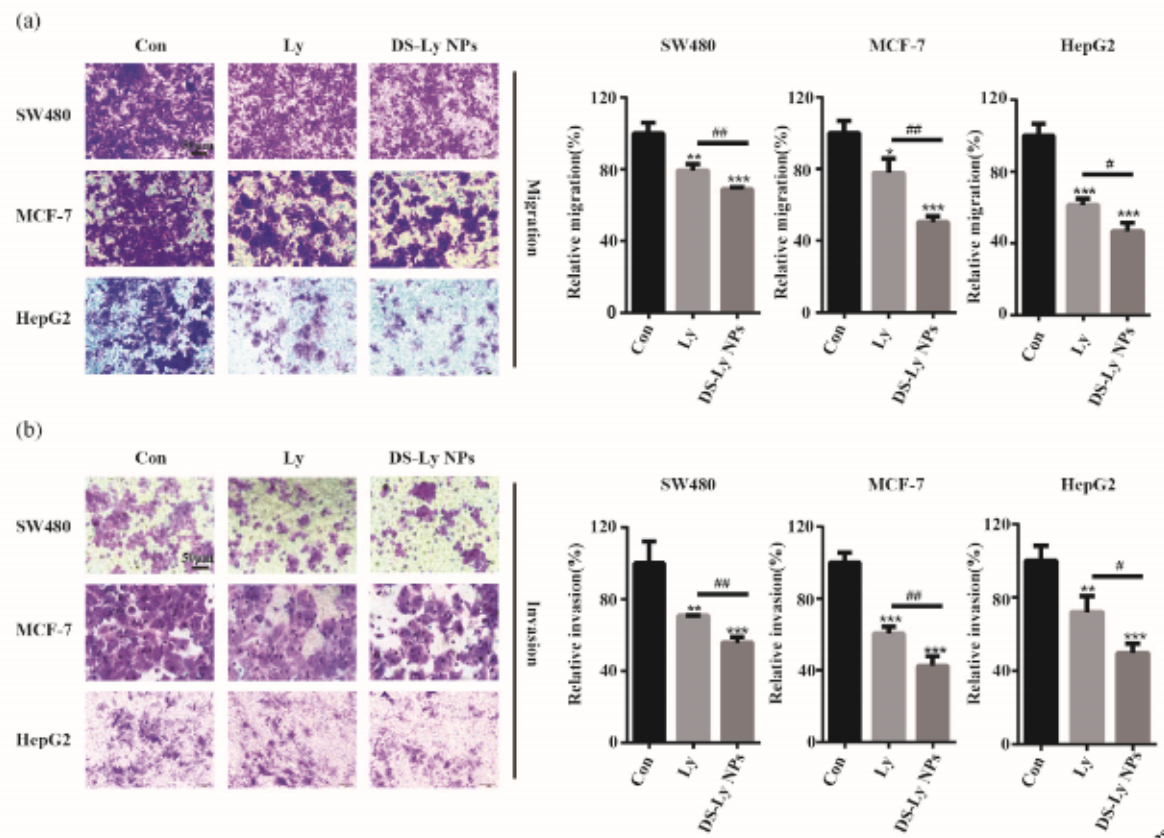

(c)
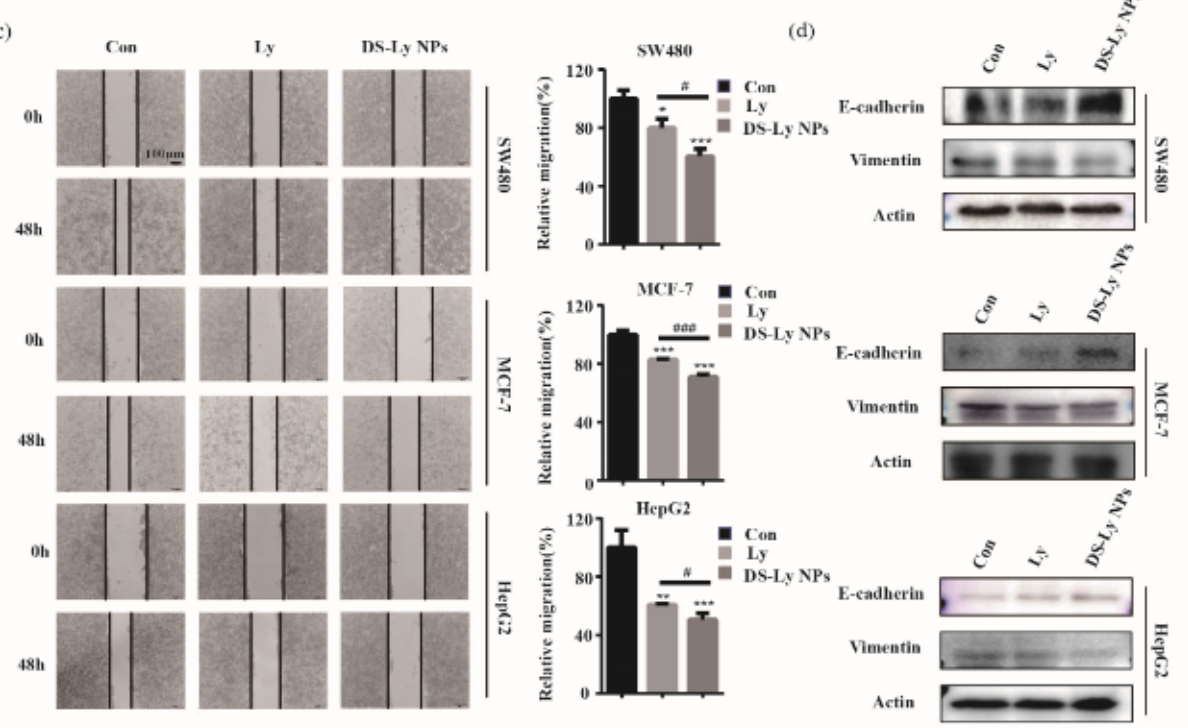

(e)

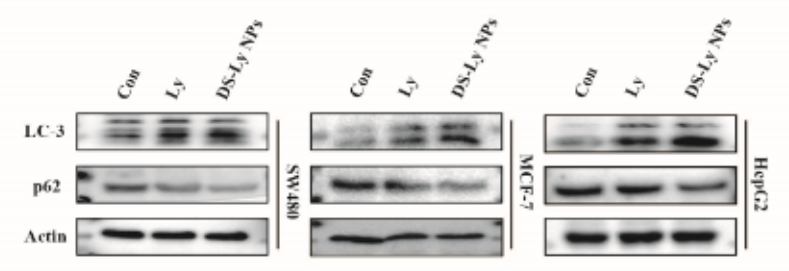

\section{Figure 4}

Ly and DS-Ly NPs suppresses the migration and promote autophagy of SW480, MCF-7 and HepG2 cells. (a) Transwell migration assays were used to determine the migratory abilities of SW480, MCF-7 and HepG2 cells and quantitative analysis. (b) Transwell invasion assays were used to detect the invasive abilities of SW480, MCF-7 and HepG2 cells and quantitative analysis. (c) Wound healing assays were Loading [MathJax]/jax/output/CommonHTML/fonts/TeX/fontdata.js F-7 and HepG2 cells and quantitative analysis. 
(d) The protein levels of E-cadherin and Vimentin were determined by western blot assays. (e) The protein levels of LC-3 and p62 were determined by western blot assays. ${ }^{\star} p<0.05 ; * \star p<0.01$; ${ }^{* \star} p<0.001$ compared to Con. \#p $<0.05, \# \# p<0.01, \# \# \# p<0.001$ compared to control treated with Ly (Student's ttest).

Figure 5

(a)

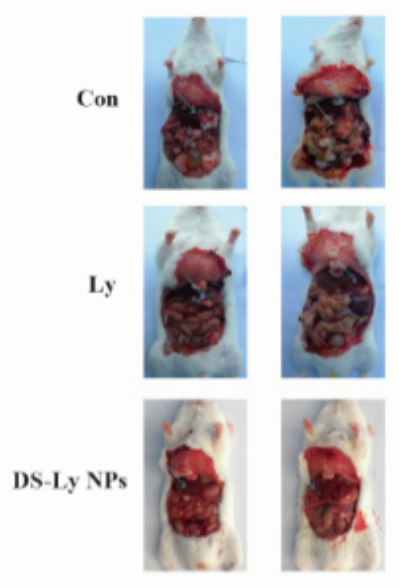

(e)

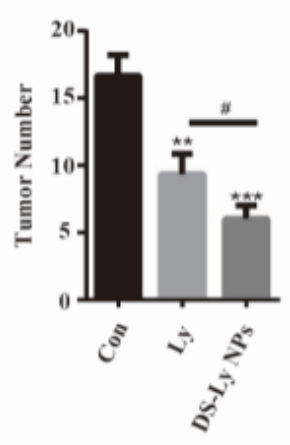

(b)

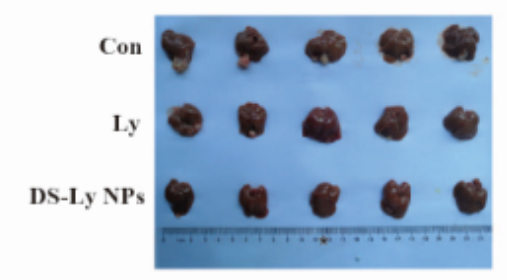

(c)

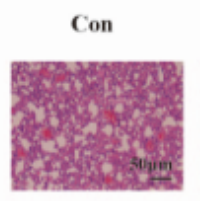

(f) (d)

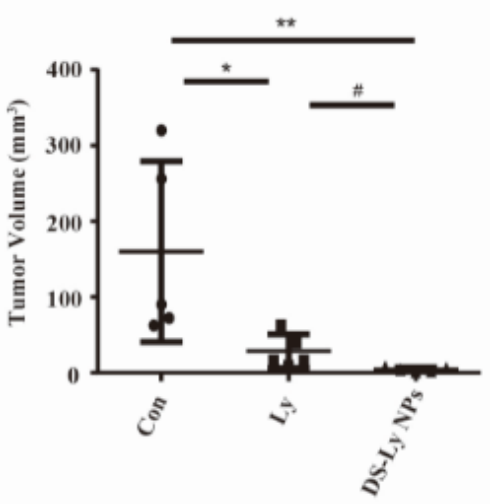

(g)

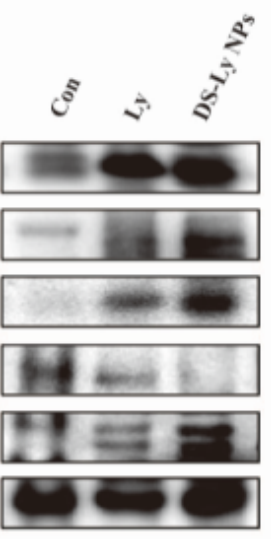

(h)

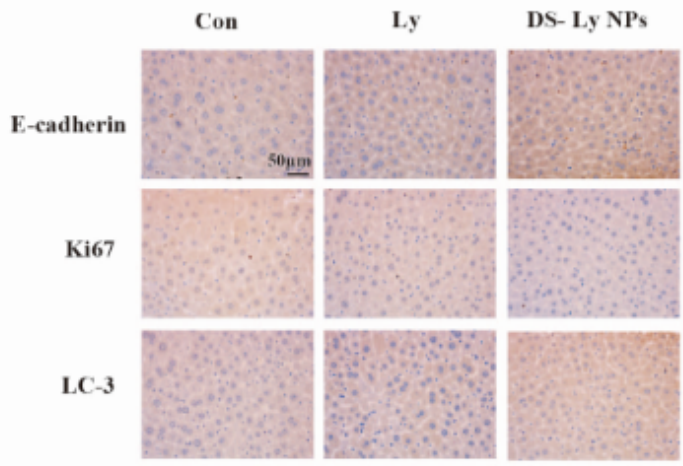

(i)
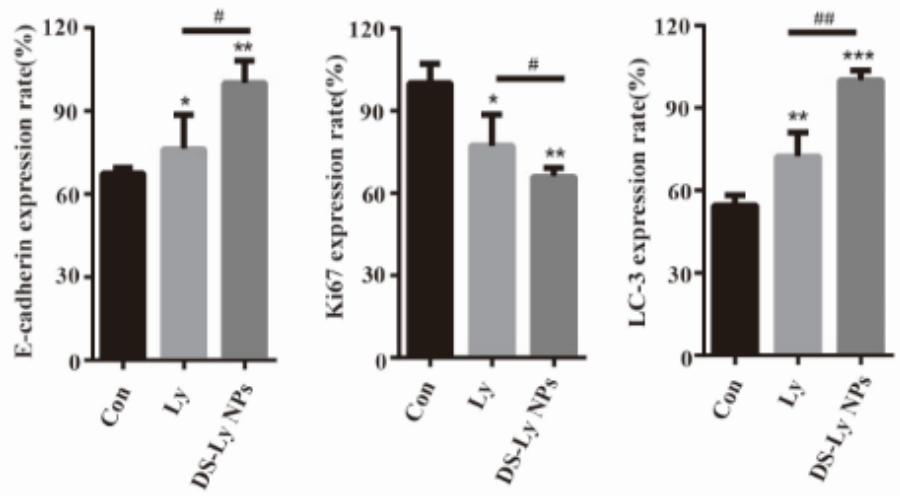

Figure 5 
Ly and DS-Ly NPs inhibits growth and development in an orthotopic mouse model of HCC. (a)-(b) The image of livers with tumor. (c) Lung metastasis of HCC was measured by H\&E staining, (e) quantification of the number of lung metastatic tumors. (d) The tumor volumes were measured. (f) The weight of the mice was measured. (g) Cleaved caspase-3, E-cadherin, Bax, p62 and LC-3 levels were determined in orthotopic tumors by western blot assays. (f) E-cadherin, Ki67 and LC-3 expressions were determined by IHC staining in orthotopic tumors, (i) E-cadherin, Ki67 and LC-3 expression rate. ${ }^{\star} p<0.05$; ${ }^{\star \star} p<0.01$; ${ }^{* \star} \mathrm{p}$ $<0.001$ compared to Con. \#p $<0.05, \# \# p<0.01$ compared to control treated with Ly (Student's t-test).

Figure 6

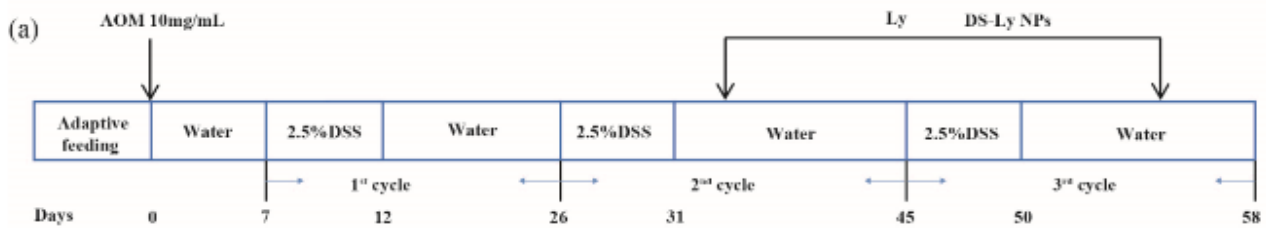

(b)

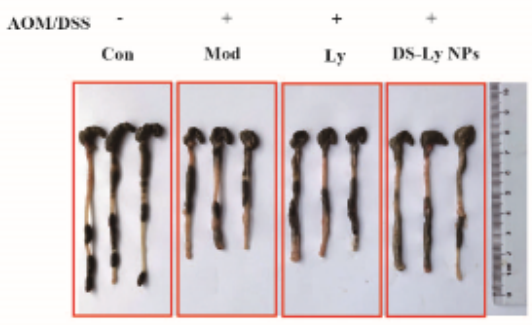

(c)

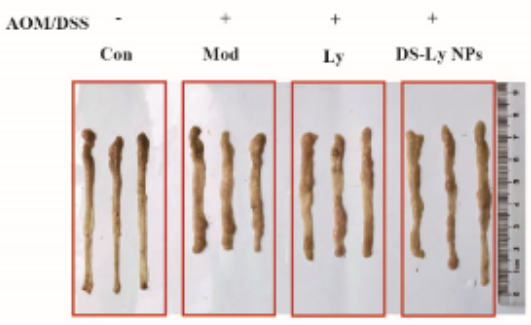

(e)

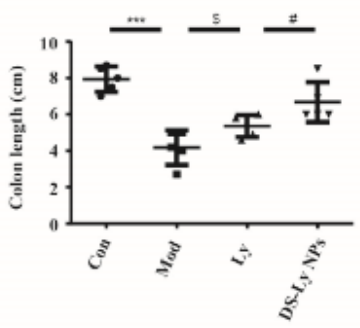

(f)

(d)

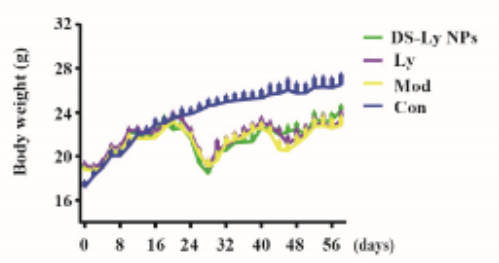

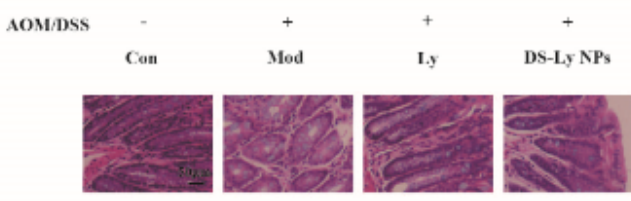

(g)

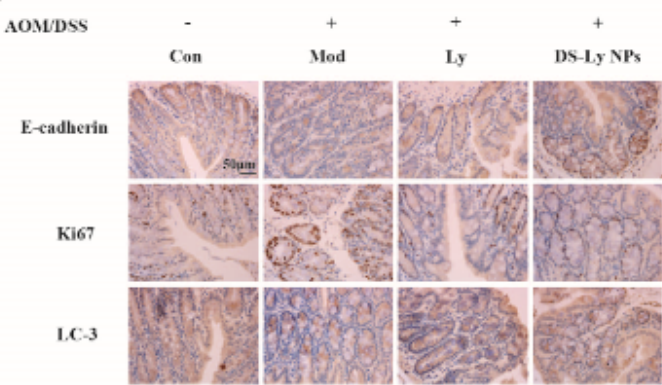

(h)

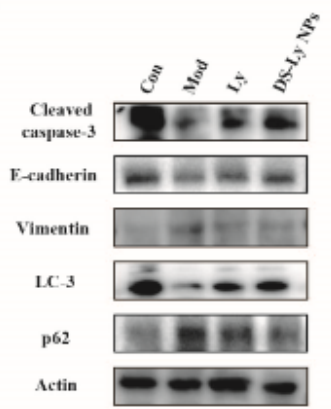

(i)

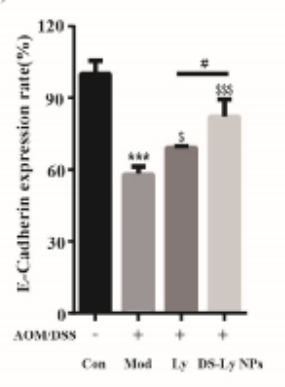

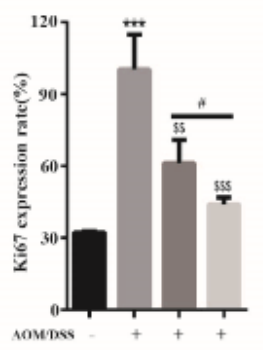

Con Mas L, Ds-Ly NP,

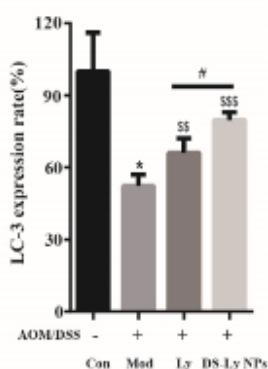

Can Mad Lo bsty 


\section{Figure 6}

Ly and DS-Ly NPs inhibits growth and development in AOM/DSS induced colorectal cancer. (a) Experimental methods for construction of the mice model of colorectal cancer. (b) Representative photograph of colons. (c) The image of colons with tumor volume and numbers. (d) Representative the colon lengths induced by AOM/DSS. (e) H\&E staining of colon. (f) The change of weight. (g) E-cadherin, Ki67 and LC-3 expressions were determined by IHC staining in colon tumor, (i) E-cadherin, Ki67 and LC-3 expression rate. (h) Cleaved caspase-3, E-cadherin, Vimentin, p62 and LC-3 levels were determined in orthotopic tumors by western blot assays. ${ }^{*} p<0.05 ; * \star * p<0.001$ compared to Con. $\# p<0.05$ compared to control treated with Ly. $\$ p<0.05$,

$$
p<0.01
$$

$\$ p<0.001$ compared to Mod.

\section{Supplementary Files}

This is a list of supplementary files associated with this preprint. Click to download.

- renamed5bb7a.docx 